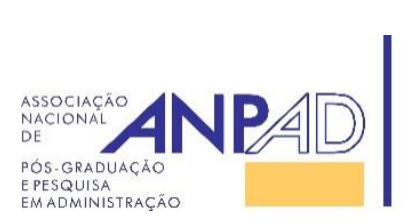

Disponível em

http://www.anpad.org.br/rac

RAC, Rio de Janeiro, v. 21, n. 2, art. 6, pp. 249-268, Mar./Abr. 2017

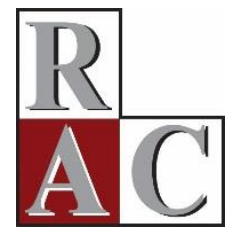

\title{
A Mediação da Ambidestria do Vendedor na Relação entre Estresse e Desempenho
}

The Mediating Role of Ambidextrous Salespeople in the Relationship between Stress and Performance

Valter Afonso Vieira ${ }^{1}$ Marco Aurelio Garcia Rosa ${ }^{1}$ Valter da Silva Faia ${ }^{1}$

Universidade Estadual de Maringá ${ }^{1}$

Artigo recebido em 18.12.2015. Última versão recebida em 25.06.2016. Aprovado em 03.07.2016. Publicado online em 26.09.2016. 


\title{
Resumo
}

Neste artigo sugerimos que a ambidestria do funcionário medeia o efeito negativo do estresse de trabalho no desempenho com vendas. Nós ampliamos a literatura sobre ambidestria, não mais posicionando-a como mediadora dos efeitos já conhecidos do contexto, do desenvolvimento do produto, da flexibilidade estratégica e da natureza dinâmica do ambiente, mas sim como mediadora dos efeitos do estresse sobre o desempenho. Um levantamento foi realizado com 307 funcionários de linha de frente que vendem produtos e serviços e gerenciam carteiras de clientes em instituições bancárias. Os achados mostraram que a capacidade do funcionário em balancear as atividades de provisão de serviços aos clientes e as atividades de vendas configura ambidestria, desencadeando maiores níveis de desempenho. Os resultados também demonstraram que o efeito negativo da percepção de ambiguidade de papéis sobre as medidas de desempenho (satisfação no trabalho e desempenho com vendas) é mediado pelo comportamento ambidestro do funcionário.

Palavras-chave: ambidestria; estresse; mediação; vendedor; desempenho.

\begin{abstract}
In this paper we suggest that an employee's ambidexterity mediates the negative effect of job stress on sales performance. The traditional literature posits ambidexterity as mediator in the context of product development, strategic flexibility and dynamic nature of the environment. We extend the ambidexterity literature by positing it as a mediator in the relationship between stress and sales performance. A survey was developed with 307 frontline bank employees who sell products and services and manage client accounts in banks. The findings showed that the ability of the salespeople to balance provision activities with sales services activities improves ambidexterity, which in turn has a positive impact on performance. The results also showed that the negative effect of perception of role ambiguity about performance measurement (work satisfaction and sales) is mediated by a salesperson's ambidextrous behavior.
\end{abstract}

Key words: ambidextrous; stress; mediating; salespeople; performance. 


\section{Introdução}

Para Raisch, Birkinshaw, Probst e Tushman (2009), o comportamento ambidestro significa a capacidade que alguns indivíduos desenvolvem para lidar com atividades aparentemente antagônicas ou por vezes complementares que exigem recursos cognitivos e afetivos. Partindo do princípio da necessidade de se atingir metas, funcionários ambidestros realizam atividades distintas em busca de objetivos múltiplos, tais como atividades que visam a eficiência por meio das rotinas e a eficácia por meio de procedimentos não rotineiros (Mom, Van Den Bosch, \& Volberda, 2009). Nesse contexto, dado o objetivo de venda pré-estabelecido, as atividades por vezes conflitantes que os vendedores desempenham tendem a ser benéficas para atingir os resultados de desempenho.

Entretanto, o excessivo volume de tarefas rotineiras e não rotineiras pode levar o funcionário a vivenciar o estresse de papel (Katz \& Kahn, 1966), que pode ser visto como a consequência da disparidade entre a percepção do funcionário sobre as características de um papel específico e o que é efetivamente realizado por ele dentro da organização (Lambert \& Lambert, 2001). Quando não há integração entre as expectativas, as motivações do papel e sua efetiva execução, o desempenho tende a ser pior (Idris, 2011; Lambert \& Lambert, 2001).

Neste artigo, sugerimos que a ambidestria do funcionário medeia o efeito nocivo do estresse de papel (Yongkang, Weixi, Yalin, Yipeng, \& Liu, 2014) no desempenho com vendas. A proposta de mediação toma por base os textos de Li e Huang (2012), Gibson e Birkinshaw (2004) e Dutta (2013), ampliando para uma nova área e com novos elementos. Nós estendemos a literatura não mais posicionando a ambidestria como mediadora dos efeitos contextuais (Gibson \& Birkinshaw, 2004), do desenvolvimento do produto (Li \& Huang, 2012), da flexibilidade estratégica (Kortmann, Gelhard, Zimmermann, \& Piller, 2014) e da natureza dinâmica do ambiente (Dutta, 2013), mas sim como mediadora dos efeitos do estresse sobre o desempenho. Ademais, nós também estendemos a literatura mensurando a ambidestria não mais em nível organizacional, conforme Gibson e Birkinshaw (2004), mas em nível individual e aplicada nas atividades de vendas e de provisão de serviços, conforme Jasmand, Blazevic e Ruyter (2012).

Por fim, com base em Idris (2011), nós sugerimos três elementos do estresse como preditores negativos do desempenho, a saber, a ambiguidade, o conflito e a sobrecarga. A tese principal do artigo é que as três dimensões de estresse interferem negativamente no desempenho por meio do efeito mediador da ambidestria. Diante do exposto, algumas lacunas são preenchidas e três contribuições para a literatura de vendas são apresentadas.

Primeiro, o papel da ambidestria como mediadora parece ter sentido na literatura, mas seu foco está ligado estritamente ao campo dos estudos organizacionais (Dutta, 2013; Kortmann et al., 2014; Li \& Huang, 2012), e não ao campo de marketing e vendas. Por exemplo, Sarkees, Hulland e Prescott (2010) encontraram uma relação positiva entre estratégias ambidestras e satisfação do cliente mediada pela implementação da ambidestria. Gibson e Birkinshaw (2004) investigaram a ambidestria por um contexto organizacional e encontraram suporte para a mediação na relação entre fatores contextuais da organização e desempenho. Outros autores também analisaram a ambidestria sob a perspectiva organizacional (Dutta 2013; Kortmann et al., 2014; Li \& Huang 2012). Não obstante, esta pesquisa amplia a discussão da ambidestria como um mecanismo mediador no contexto individual de vendas, investigando funcionários de vendas das instituições financeiras e encontrando suporte para as hipóteses sugeridas. Essa perspectiva individual está em linha com Jasmand et al. (2012), Yu, Patterson e Ruyter (2013, 2015) e Patterson, Yu e Kimpakorn (2014), mostrando a capacidade do vendedor em combinar vendas com o serviço de atendimento ao cliente.

Segundo, o trabalho estende a discussão do estresse no ambiente de trabalho para três dimensões, sendo elas a ambiguidade de papéis, o conflito de papéis e a sobrecarga de papéis (Idris 2011). Pouco se sabe sobre o real impacto de cada dimensão isoladamente no desempenho do vendedor. Sendo assim, avançamos nos achados de Rizzo, House e Lirtzman (1970), sobre o estresse gerado pelo conflito, e nas considerações de Thiagarajan, Chakrabarty e Taylor (2006), sobre sobrecarga no trabalho. Os dados da 
pesquisa mostraram que os efeitos das três dimensões de estresse no desempenho do vendedor são prejudiciais para o desempenho. A ambiguidade se mostrou mais relevante ao explicar negativamente $o$ desempenho.

Terceiro, ampliamos a capacidade de mensurar o desempenho do vendedor por meio de duas perspectivas diferentes, sendo elas o desempenho subjetivo em vendas e a satisfação no trabalho. A primeira perspectiva está relacionada aos resultados das metas com vendas, e a segunda está associada à qualidade de vida no trabalho do funcionário. Os achados do trabalho são congruentes com os resultados de Verhoef e Leeflang (2009) e Jasmand et al. (2012).

A Tabela 1 apresenta uma comparação entre este artigo e outros textos relacionados ao tema. É possível notar a contribuição deste trabalho ao analisar o efeito mediador da ambidestria na área de vendas, assim como ao investigar o efeito indireto do estresse no trabalho sobre medidas de desempenho.

Tabela 1

\section{Comparação com a Literatura Existente}

\begin{tabular}{|c|c|c|c|c|}
\hline Autores & Foco Principal & $\begin{array}{l}\text { Ambidestria } \\
\text { Individual }\end{array}$ & $\begin{array}{c}\text { Ambidestria } \\
\text { Organizacional }\end{array}$ & $\begin{array}{l}\text { Estresse de } \\
\text { Papel }\end{array}$ \\
\hline Jasmand et al. (2012) & $\begin{array}{l}\text { Testa a relação antecedente da ambidestria } \\
\text { em termos do modo regulatório do } \\
\text { funcionário, da identificação com o time e } \\
\text { dos processos padronizados. Testa os efeitos } \\
\text { da ambidestria em medidas de desempenho, } \\
\text { contudo, não testa o efeito indireto. }\end{array}$ & Sim & & \\
\hline $\begin{array}{l}\text { Yu et al. (2013, } \\
\text { 2015) }\end{array}$ & $\begin{array}{l}\text { Testa a relação antecedente da ambidestria } \\
\text { em termos de empoderamento, suporte do } \\
\text { time e da equipe, liderança transformacional, } \\
\text { autoeficácia e orientação aos resultados. }\end{array}$ & Sim & & \\
\hline $\begin{array}{l}\text { Patterson et al. } \\
\text { (2014) }\end{array}$ & $\begin{array}{l}\text { Testa a relação antecedente da ambidestria } \\
\text { em termos de autoeficácia, relação líder- } \\
\text { liderado e clima organizacional, moderada } \\
\text { pela experiência do funcionário e pelo } \\
\text { dinamismo do ambiente. }\end{array}$ & Sim & & \\
\hline $\begin{array}{l}\text { Gibson e Birkinshaw } \\
(2004)\end{array}$ & $\begin{array}{l}\text { Testa o efeito mediador da ambidestria no } \\
\text { nível organizacional na relação entre a gestão } \\
\text { da performance e contexto social e o } \\
\text { desempenho da unidade de negócio. }\end{array}$ & & Sim & \\
\hline Idris (2011) & $\begin{array}{l}\text { Testa o efeito direto da sobrecarga, da } \\
\text { ambiguidade e do conflito de papéis na } \\
\text { tensão individual. }\end{array}$ & & & Sim \\
\hline Li e Huang (2012) & $\begin{array}{l}\text { Testa o efeito mediador da ambidestria na } \\
\text { relação entre proficiência no } \\
\text { desenvolvimento de produtos e desempenho } \\
\text { dos novos produtos. }\end{array}$ & & Sim & \\
\hline Dutta (2013) & $\begin{array}{l}\text { Testa o efeito mediador da ambidestria na } \\
\text { relação entre dinamismo do ambiente, } \\
\text { contexto organizacional e renovação } \\
\text { estratégica. }\end{array}$ & & Sim & \\
\hline Sarkees et al. (2010) & $\begin{array}{l}\text { Testa o efeito mediador da implementação da } \\
\text { função de marketing na relação entre } \\
\text { ambidestria e desempenho organizacional. }\end{array}$ & & Sim & \\
\hline
\end{tabular}


Tabela 1 (continuação)

\begin{tabular}{|c|c|c|c|c|}
\hline Autores & Foco Principal & $\begin{array}{l}\text { Ambidestria } \\
\text { Individual }\end{array}$ & $\begin{array}{c}\text { Ambidestria } \\
\text { Organizacional }\end{array}$ & $\begin{array}{c}\text { Estresse de } \\
\text { Papel }\end{array}$ \\
\hline $\begin{array}{l}\text { Kortmann et al. } \\
\text { (2014) }\end{array}$ & $\begin{array}{l}\text { Testa o efeito mediador da ambidestria na } \\
\text { relação entre flexibilidade estratégica e } \\
\text { eficiência operacional. }\end{array}$ & & Sim & \\
\hline $\begin{array}{l}\text { Yongkang, Weixi, } \\
\text { Yalin, Yipeng e Liu } \\
\text { (2014) }\end{array}$ & $\begin{array}{l}\text { Testa o efeito direto da sobrecarga, da } \\
\text { ambiguidade e do conflito de papéis sobre o } \\
\text { estresse, a ansiedade e a pressão no trabalho. }\end{array}$ & & & Sim \\
\hline Esta pesquisa & $\begin{array}{l}\text { Testa o efeito mediador da ambidestria no } \\
\text { nível individual na relação entre sobrecarga, } \\
\text { ambiguidade e conflito de papéis e } \\
\text { desempenho com vendas e satisfação no } \\
\text { trabalho. }\end{array}$ & Sim & & Sim \\
\hline
\end{tabular}

Após essa breve introdução, o artigo está estruturado conforme apresentado a seguir. No referencial teórico, discute-se a ambidestria e as três dimensões do estresse de trabalho. Na sequência, um framework teórico é proposto, no qual a ambidestria possui um papel mediador na relação entre o estresse e desempenho. Posteriormente, foram apresentados os procedimentos metodológicos da pesquisa de campo realizada com 307 funcionários da linha de frente que vendem produtos e serviços financeiros. Por fim, os resultados são discutidos e as considerações finais são apresentadas.

\section{Referencial Teórico}

\section{Comportamento ambidestro}

O conceito seminal sobre ambidestria foi discutido por Duncan (1974) ao propor um modelo que divide o processo de criação de uma organização inovadora em duas etapas. A primeira fase, iniciação, exige maior grau de complexidade, menor grau de formalização e baixa centralização, ao passo que a segunda fase, implantação, é caracterizada por alto nível de formalização, baixo nível de complexidade e alto nível de centralização (Duncan, 1974). Nesse contexto, a ambidestria se refere à busca simultânea pela organização de dois objetivos estratégicos, sendo iniciação e implantação (Simsek, 2009).

Para March (1991), as atividades de iniciação envolvem a exploração de novas competências e estão diretamente vinculadas a mudanças, decisões envolvendo riscos, flexibilidade, experimentação e criação de novos produtos. Contrariamente, as atividades de implantação envolvem a explotação de competências atuais e estão ligadas ao refinamento, à escolha eficiente, à produção e à execução (Gibson \& Birkinshaw, 2004). Para March (1991), na busca por esses dois objetivos, o principal foco de atenção de uma organização está em aproveitar os conhecimentos já existentes, buscar novos conhecimentos e manter um balanceamento adequado entre essas ações (ambidestria). Esses dois conjuntos de atividades são díspares e não substituíveis, por isso as organizações buscam uma complementaridade executando ambos os elementos de modo balanceado (Gibson \& Birkinshaw, 2004). A capacidade da organização em explorar novas competências e competências atuais simultaneamente (Gilsing, 2002) está diretamente vinculada à sua capacidade de absorver conhecimentos externos e implementá-los (Jansen, Bosch, \& Volberda, 2006).

É possível uma reflexão sobre a transposição da ambidestria do nível organizacional para o nível individual, uma vez que a exploração de competências novas e atuais ocorre devido à assimilação de aprendizado e às práticas de ações executadas pelo elemento humano (Benner \& Tushman, 2003). Estudos em nível organizacional assumem a existência de uma homogeneidade no nível individual, negligenciando como os membros das organizações podem influenciar a habilidade da firma na busca por objetivos conflitantes (Bonesso, Gerli, \& Scapolan, 2014). No entanto, o sucesso para o 
gerenciamento dos conflitos da ambidestria reside em variáveis contextuais que permitem e encorajam os funcionários a fazerem seus próprios julgamentos sobre como dividir seu tempo entre as demandas conflitantes na busca do alinhamento e da adaptabilidade (Gibson \& Birkinshaw, 2004).

Portanto, a ambidestria passa a ser característica da organização como um todo, a partir das ações de cada um dos seus indivíduos (Gibson \& Birkinshaw, 2004). Em outras palavras, a ambidestria individual pode ser o estágio inicial de fomento à ambidestria organizacional. Para que a ambidestria individual possa ocorrer de forma favorável à organização, é crucial a construção de um contexto organizacional que facilite a implementação de ações e atitudes dos funcionários de vendas de modo bem sucedido (Gibson \& Birkinshaw, 2004; Yu, Patterson, \& Ruyter, 2013). Variáveis contextuais influenciam a atitude e o comportamento dos funcionários e, assim, determinam a capacidade da organização de buscar estratégias conflitantes (Yu et al., 2013).

Diante do apresentado, as características dos funcionários constituem aspectos que devem ser observados, pois podem influenciar significativamente a ambidestria individual e ocasionar maiores níveis de desempenho (Jasmand, Blazevic, \& Ruyter, 2012). Por exemplo, o desejo do funcionário de adquirir novas habilidades e competências, para enfrentar novas situações e para adquirir novos conhecimentos, conduz a diferentes graus de aprendizado que, por sua vez, interferem positivamente no nível de ambidestria individual (Yu, Patterson, \& Ruyter, 2015). Do mesmo modo, quanto maior for a crença do funcionário na sua própria capacidade de realizar tarefas, denominada autoeficácia, maior tende a ser também o nível de ambidestria individual (Patterson, Yu, \& Kimpakorn, 2014; Yu et al., 2015).

\section{Estresse de papel}

O estresse no trabalho pode ser compreendido como um estado permanente de esforço mental que resulta em tensões psicológicas negativas que afetam o desempenho do indivíduo (Aghdasia, Kiamaneshb, \& Ebrahim, 2011). Possivelmente, a partir dos estudos realizados por Kahn, Wolfe, Quinn e Snoek (1964), o estresse proveniente das atividades organizacionais ganhou maior notoriedade nas pesquisas em administração, resultante principalmente do grande desafio encontrado pelas organizações em melhorar a qualidade do serviço e em manter a satisfação dos funcionários com o ambiente de trabalho.

Considerando que o contexto de ambidestria exige o desempenho de tarefas múltiplas (Duncan, 1974), é coerente esperar que as três dimensões do estresse de papel possam aparecer com frequência no dia a dia dos funcionários da linha de frente, exercendo, de alguma forma, influência negativa no desempenho. Nesse sentido, esta pesquisa se propõe a colaborar com os estudos sobre estresse (Idris, 2011; Posig \& Kickul, 2003; William \& Alliger, 1994) buscando evidências empíricas dos efeitos das três dimensões de estresse no desempenho do funcionário por meio do comportamento ambidestro. A seguir, as três dimensões são detalhadas.

O conflito de papel ocorre quando o funcionário se confronta com expectativas de papéis que, a seu ver, são incompatíveis com o status social que ocupa (Yongkang et al., 2014), ou quando as pessoas discordam sobre quais são as expectativas para uma determinada função (Muchinsky, 2000). O conflito de papel é o descompasso percebido entre os requisitos e as expectativas dos sujeitos da equipe de vendas (Verbeke, Dietz, \& Verwaal, 2011). Esse conflito pode ser percebido durante a realização das tarefas ligadas à execução do papel quando há possivelmente uma incongruência entre as expectativas de dois ou mais funcionários em relação à tarefa solicitada.

A ambiguidade de papel ocorre quando os funcionários sentem que eles não possuem informações suficientes para executar de forma eficaz suas tarefas e quando eles estão incertos sobre as expectativas dos parceiros da equipe quanto às funções (Verbeke et al., 2011). A ambiguidade dos funcionários de vendas é tratada como o grau em que uma informação necessária ao exercício da função está faltando e como a incerteza que o funcionário tem sobre as expectativas dos demais membros da equipe com relação a ele, fatos que, por sua vez, tendem a apresentar uma relação negativa com o desempenho (Yongkang et al., 2014). 
A sobrecarga de papel é o excedente percebido da demanda de trabalho quando comparado com a motivação e com as habilidades pessoais (Verbeke et al., 2011). Em outras palavras, o funcionário pode sentir a sobrecarga de papel quando percebe que há muitas responsabilidades ou muitas atividades para executar, considerando o tempo disponível, a motivação, as habilidades e outras restrições. Essa sobrecarga de papel tende a dificultar o comportamento ambidestro possivelmente devido ao acúmulo de atividades a serem resolvidas.

\section{Hipóteses e Modelo Conceitual}

Os trabalhos de Behrman e Perreault (1982) e Verbeke, Dietz e Verwaal (2011) mostram uma clara relação inversa entre o estresse e o desempenho em vendas. O estresse no trabalho é a reação do corpo diante de um elemento estressor, que pode ser percebido como favorável ou não, ou seja, alguns elementos do trabalho como a sobrecarga ou a incongruência de funções podem ser vistos, em um primeiro momento, como desafiadores (Le Fevre, Matheny, \& Kolt, 2003). Contudo, em altos níveis ou em um grande período de exposição, esses elementos podem produzir reações psicológicas e fisiológicas negativas (Yongkang et al., 2014).

No campo da saúde, Chida, Hamer, Wardle e Steptoe (2008) e Heikkilä et al. (2013) mostraram em estudos meta-analíticos que o estresse pode até mesmo causar alguns tipos de câncer. Desse modo, os mecanismos que sugerem o estresse como um preditor negativo do desempenho podem ir desde problemas psicológicos, psiquiátricos, psicossociais, disfunções neurológicas, hipertensão, etc., até estados emocionais como ansiedade e depressão (Ford, Cerasoli, Higgins, \& Decesare, 2011), que prejudicam a capacidade de desempenhar tarefas. Diante do exposto, tem-se que:

$\mathbf{H}_{1 \mathbf{a}}$ : O estresse de conflito do vendedor tem efeito negativo no desempenho com vendas.

$\mathbf{H}_{\mathbf{1 b}}$ : $\mathrm{O}$ estresse de ambiguidade do vendedor tem efeito negativo no desempenho com vendas.

$\mathbf{H}_{1 \mathbf{c}}$ : $\mathrm{O}$ estresse de sobrecarga do vendedor tem efeito negativo no desempenho com vendas.

Além da redução do bem-estar físico (Heikkilä et al., 2013), a queda do desempenho do funcionário pode se dar também em função da diminuição dos índices de satisfação no trabalho (Kahn, Wolfe, Quinn, \& Snoek, 1964). Propõe-se que os três fatores do estresse também explicam negativamente a satisfação no trabalho, prejudicando a capacidade do funcionário de estar bem com a atividade no dia a dia. A cobrança por tarefas conflitantes, a falta de informações para o trabalho e a sobrecarga de atividades constituem elementos estressores que tendem a reduzir os níveis de satisfação com o trabalho.

A influência negativa do estresse ocorre por meio de múltiplos mecanismos, tais como sobrecarga de atividade além dos limites de recursos, o ambiente negativo entre times de trabalho, as agressões físicas ao corpo, as pressões de trabalhos extensivos (ex.: bater metas) e as atividades extras (Ahsan, Abdulah, Fie, \& Alam, 2009). Tais condições levam à fadiga e acarretam uma maior insatisfação com o trabalho, prejudicando a saúde do funcionário (Cass, Siu, Faragher, \& Cooper, 2003). Esse prejuízo da saúde física e mental pode prejudicar os níveis de satisfação com as atividades e rotinas. Portanto:

$\mathbf{H}_{1 \mathbf{d}}$ : $\mathrm{O}$ estresse de conflito do vendedor tem efeito negativo na satisfação no trabalho.

$\mathbf{H}_{1 \mathbf{e}}$ : O estresse de ambiguidade do vendedor tem efeito negativo na satisfação no trabalho.

$\mathbf{H}_{1 \mathbf{f}}$ : O estresse de sobrecarga do vendedor tem efeito negativo na satisfação no trabalho.

Sugere-se que a ambidestria do funcionário na atividade de vendas exerce um efeito positivo no desempenho por meio de dois mecanismos. O primeiro é a capacidade de realizar cross-selling como forma de alavancar o resultado. Nesse contexto, o vendedor tende a ponderar elementos que completam 
o pedido inicial do consumidor e busca oferecer produtos adjacentes que apresentem maior capacidade de gerar satisfação com o uso (Jasmand et al., 2012). Dependendo do momento da compra, a venda cross-selling, que é um elemento da ambidestria, pode ser aprimorada com a venda up-selling, gerando melhores resultados de desempenho (Günes, Aksin, Örmeci \& Özden, 2010).

O segundo mecanismo corresponde aos elementos de provisionamento de serviços. Nesse ponto, o vendedor tende a traçar estratégias de como abordar os consumidores, buscando oportunidades para oferecer produtos novos, apresentar benefícios da compra e de pós-compra, entre outros (Deery, Roderick, \& Walsh, 2002). Nessa conjuntura, as estratégias deveriam elevar o desempenho por meio da qualidade do provisionamento do serviço e do ganho em produtividade por meio do cumprimento de metas (Yu et al., 2013). Sugere-se que ambos os elementos (cross-/up-selling e provisão de serviços) fomentam o comportamento ambidestro do vendedor, o que acarreta em maior desempenho com vendas e maior satisfação com o trabalho. Diante do apresentado, as seguintes hipóteses são sugeridas:

$\mathbf{H}_{2 \mathbf{a}}$ : A ambidestria do vendedor tem efeito positivo no desempenho com vendas.

$\mathbf{H}_{2 \mathbf{b}}$ : A ambidestria do vendedor tem efeito positivo na satisfação no trabalho.

Neste tópico, as hipóteses de mediação da ambidestria do vendedor, ponto central do trabalho, são descritas. O efeito indireto do estresse via ambiguidade de papel pode ocorrer quando os funcionários sentem que eles não possuem informações suficientes e/ou claras para executar de forma eficaz suas tarefas e quando eles estão incertos (Katz \& Kahn, 1966) sobre as expectativas dos parceiros em relação às funções (Verbeke et al., 2011). Informações aos funcionários que não são claras podem gerar dificuldades na interpretação da priorização dos gerentes, podendo causar interpretações divergentes, gerando poucas expectativas de resultados futuros e suprimindo os níveis de esforço para cada atividade (Van der Borgh \& Schepers, 2014).

Nesse sentido, a falta de informações satisfatórias e/ou declaradas sobre produtos e serviços e a incerteza sobre os procedimentos para concretizar a venda podem prejudicar as atividades de provisão de serviços e de cross-/up-selling, deixando-as obscuras, gerando certo grau de dúvida no funcionário e, indiretamente, reduzindo o desempenho (Kahn et al., 1964). Por consequência, o processo de dúvida e de irresolução da ambiguidade pode diminuir o desempenho por meio da incerteza da atividade a ser desempenhada (Katz \& Kahn, 1966). Quando a percepção do funcionário é inconsistente com seus comportamentos, como as atividades de provisão de serviços e vendas, a tendência é que sua satisfação no trabalho e seus resultados sejam menores (Bonesso et al., 2014). Assim sendo, acredita-se que o estresse da ambiguidade desfavoreça o resultado com vendas e a satisfação no trabalho não apenas de forma direta, mas também de forma indireta, via redução do comportamento ambidestro. Logo:

$\mathbf{H}_{3 \mathbf{a}}$ : O Comportamento ambidestro tem papel mediador na relação negativa entre ambiguidade e desempenho (satisfação no trabalho).

$\mathbf{H}_{3 \mathrm{~b}}$ : O Comportamento ambidestro tem papel mediador na relação negativa entre ambiguidade e desempenho (desempenho com vendas).

O conflito de papel acontece quando o funcionário de linha de frente se confronta com expectativas de papéis que são incompatíveis com o status social que ocupa (Yongkang et al., 2014) ou acontece quando os funcionários discordam sobre quais são as expectativas para uma determinada função (Muchinsky, 2000). Duas argumentações podem ser úteis para explicar o efeito indireto do conflito via mediação da ambidestria. Primeiro, no caso do banco, o funcionário pode ser forçado a vender produtos e serviços para clientes endividados ou aposentados como finalidade de atingir metas e obter comissões extras. Tal processo pode ser incompatível com o status social que o funcionário da agência ocupa ou com as políticas organizacionais (Idris, 2011), gerando conflito de expectativas de papéis e confusão sobre o comportamento ambidestro a desempenhar. Esse conflito pode influenciar indiretamente o desempenho via redução do comportamento ambidestro, prejudicando (mesmo com a capacidade positiva de provisão de serviços) o resultado. Tal efeito mediado ocorre uma vez que a venda desnecessária ao cliente pode gerar uma tensão psicológica no funcionário produzida pela presença simultânea de motivos contraditórios. 
Segundo, o funcionário do banco pode estar vivenciando um conflito de papéis por ter que vender produtos ou abrir novas contas, atividades fim, e por ter que, ele mesmo, fazer o papel burocrático/documental para que tais ações sejam concretizadas, atividades meio, o que também reduz o tempo para novas vendas. Nesse caso, pode haver uma discordância entre os funcionários que vendem produtos (ex.: gerentes) e os que atuam na burocracia de processos (ex.: tesouraria). Essas discordâncias entre as atividades e a restrição de recursos (Idris, 2011) podem ser prejudiciais ao ponto de reduzirem o desempenho das ações de ambidestria do funcionário (ex.: priorizar um grupo de atividades em detrimento do outro), diminuindo, consequentemente, o desempenho e a satisfação no trabalho. Portanto, o conflito tende a ter um efeito indireto sobre o desempenho via mediação do comportamento ambidestro. Assim sendo:

$\mathbf{H}_{4 a}$ : O comportamento ambidestro tem papel mediador na relação negativa entre conflito de papéis e desempenho (satisfação no trabalho).

$\mathbf{H}_{\mathbf{4}}$ : O comportamento ambidestro tem papel mediador na relação negativa entre conflito de papéis e desempenho (desempenho com vendas).

A sobrecarga de papéis representa o volume de exigências inerentes a um dado papel (Claessens, Van Eerde, Rutte, \& Roe, 2004) e ocorre quando as expectativas de papel são muito superiores às capacidades que o funcionário acredita ter e a motivação que sente para cumpri-las (Singh, 1998). Em vista disso, a sobrecarga de papéis tende a elevar o estresse no ambiente de trabalho, podendo desmotivar o empregado para determinadas ações (Pinheiro \& Günther, 2002). A sobrecarga de papéis para o funcionário de linha de frente pode ocorrer quando há inúmeras metas e atividades a serem cumpridas com um tempo muito curto (sem expectativa de hora extra) e com pressões por resultados, gerando estresse.

Mesmo o funcionário tendo autonomia para decidir quanto à melhor forma de aplicar os recursos nas atividades conflitantes (Jasmand et al., 2012; Yu et al., 2013), a escassez de recursos pode levá-lo a priorizar uma atividade ou outra conforme o senso de urgência. Devido às múltiplas obrigações, deveres e tarefas, que aumentam o desgaste e a sobrecarga do empregado (Thiagarajan, Chakrabarty, \& Taylor, 2006), o impacto positivo do comportamento ambidestro sobre o desempenho tende a ser negativo. Nesse sentido, o estresse de sobrecarga de papéis pode reduzir diretamente o comportamento ambidestro, prejudicando a capacidade mental do colaborador de realizar múltiplas atividades, o que reduz, indiretamente, o desempenho com vendas e a satisfação no trabalho. Portanto:

$\mathbf{H}_{5 \mathbf{a}}$ : O comportamento ambidestro tem papel mediador na relação negativa entre sobrecarga de papéis e desempenho (satisfação no trabalho).

$\mathbf{H}_{5 \mathbf{b}}$ : O comportamento ambidestro tem papel mediador na relação negativa entre sobrecarga de papéis e desempenho (desempenho com vendas).

A Figura 1 apresenta o modelo teórico do trabalho, que foi examinado com dados de funcionários de linha de frente que vendem produtos e serviços nos bancos. O comportamento ambidestro, mensurado pela perspectiva de provisão de serviços e de cross-/up-selling, medeia a relação negativa entre estresse e resultados. As covariáveis corrigem os níveis de variância do desempenho. 


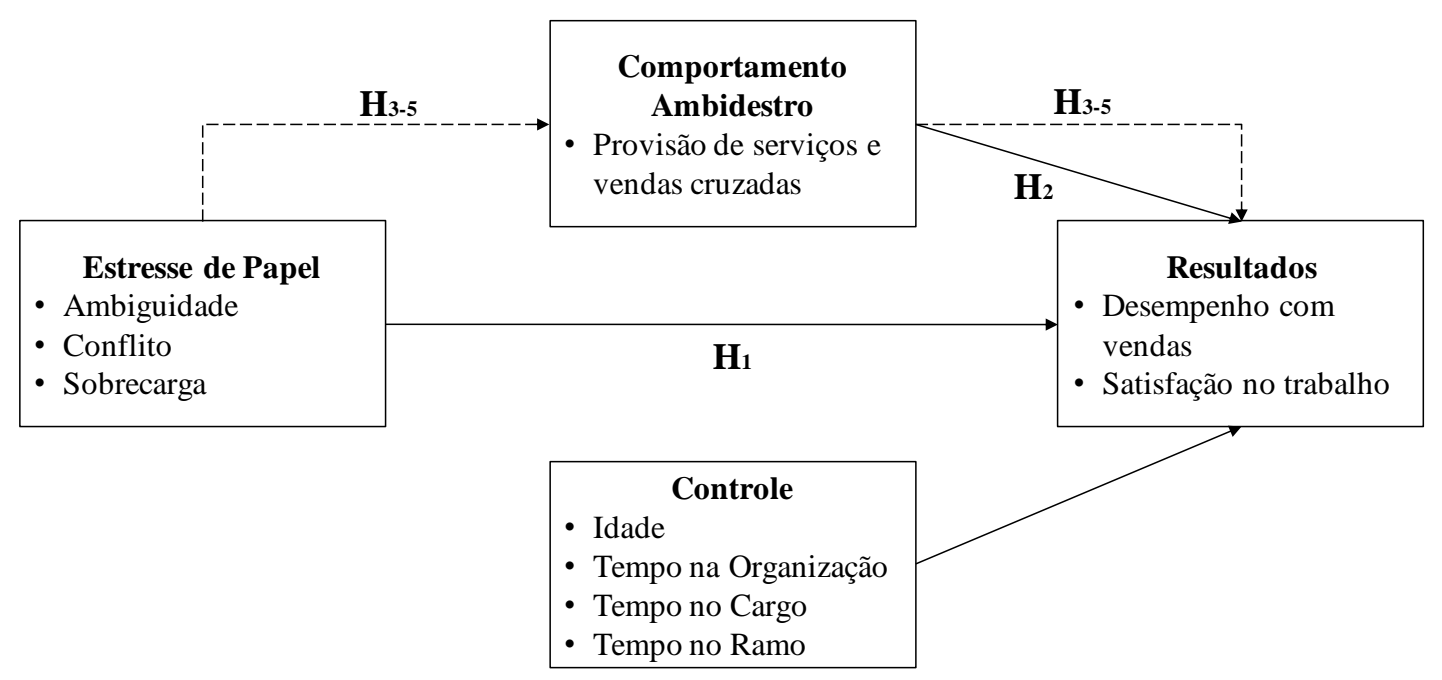

Figura 1. Modelo da Mediação do Comportamento Ambidestro

Na figura 1, as setas pontilhadas significam relações mediadas; as setas contínuas, relações diretas.

\section{Procedimentos Metodológicos}

\section{Design de pesquisa}

A pesquisa foi feita com funcionários de instituições financeiras, semelhante aos trabalhos de $\mathrm{Yu}$ et al. (2013, 2015) e Patterson et al. (2014). Ao entrar em contato com os clientes, o funcionário do banco tem a missão de resolver os problemas destes em relação aos produtos contratados, enquanto procura uma nova oportunidade de venda do tipo cross-/up-selling. Os funcionários devem tirar dúvidas quanto a investimentos, conta corrente, empréstimos, seguros, cartões, entre outros serviços já contratados, ao mesmo tempo em que devem almejar novas vendas guiados por diversas metas. Essa busca simultânea em prestar um serviço de atendimento junto com a busca por metas de vendas requer que o funcionário gerencie exigências comportamentais diferentes, compreendendo, assim, a concepção do comportamento ambidestro. Ademais, a habilidade do funcionário de procurar oportunidades de vendas enquanto presta serviços de qualidade aos clientes é uma característica importante nos bancos. Ter um grupo de funcionários ambidestros provoca melhoria nas vendas e na redução dos custos (Yavas, Karatepe, Avci, \& Tekinkus, 2003).

Diante desse contexto, o comportamento ambidestro requer engajamento do funcionário para gerenciar demandas de tarefas, ora complementares, ora conflitantes (Bonesso et al., 2014), gerando um grande desafio, já que deve perseguir múltiplos objetivos. Paralelamente à busca por tais objetivos, há a possibilidade da presença de estressores de papel que afetam indiretamente a relação entre ambidestria e os resultados esperados.

\section{Coleta de dados}

Para a coleta de dados, utilizou-se um questionário estruturado que foi entregue pessoalmente nas agências bancárias com a autorização do gerente da agência e com um termo de compromisso que apontava a garantia de anonimato e que descrevia os objetivos do estudo e a finalidade científica dos dados coletados. Para a elaboração do questionário, inicialmente uma validade de face (Nevo, 1985) foi realizada objetivando entendimentos e correções necessárias sobre as afirmativas. Três professores de marketing, especialistas na área de vendas, participaram desse processo. As escalas utilizadas originaram-se de trabalhos desenvolvidos em língua estrangeira e, por isso, foram submetidas à técnica de tradução reversa. 
Após um período de 30 dias e diversos follows-up, o pesquisador buscou os questionários para posterior tabulação. Os respondentes eram funcionários de linha de frente que gerenciavam carteiras de clientes em instituições bancárias. Houve um total de 317 respondentes. Após o tratamento e as análises dos dados omissos, optou-se pela retirada de 10 questionários com respostas incorretas e erros, gerando um montante final de 307 respondentes.

\section{Definição e mensuração das variáveis}

A ambidestria é estudada no âmbito do comportamento do funcionário da linha de frente. $\mathrm{O}$ comportamento ambidestro reflete o engajamento do funcionário em termos de provisão de serviços e cross-/up-selling durante os encontros com o cliente (Jasmand et al., 2012). A mensuração do comportamento ambidestro foi realizada a partir da escala desenvolvida por Jasmand et al. (2012), sendo composta por 12 itens agrupados em duas dimensões: provisão de serviços e cross-/up-selling. $\mathrm{O}$ conflito de papel é compreendido como a incompatibilidade entre as expectativas e demandas associadas ao papel a ser desempenhado pelo funcionário (Idris, 2011). Essa dimensão do estresse foi mensurada pela escala apresentada por Rizzo et al. (1970), que consiste em 8 itens. A ambiguidade de papel é abrangida como a incerteza sobre o que o indivíduo responsável por uma atividade específica deveria fazer, ou seja, a falta de definições acerca das responsabilidades, expectativas e comportamentos de um indivíduo em um determinado cargo (Rizzo, House, \& Lirtzman, 1970). Para mensurar a ambiguidade de papel, foi utilizada uma escala de 6 itens desenvolvida por Rizzo et al. (1970). A sobrecarga de papéis representa o volume de exigências inerentes a um dado papel e ocorre quando as expectativas do papel são muito superiores às capacidades e motivações que o indivíduo acredita ter para desempenhá-las, enfrentado múltiplas obrigações, deveres e tarefas, e considerando que não é possível realizá-las em tempo hábil (Thiagarajan et al., 2006). A escala de sobrecarga de papel, composta por 5 itens, foi adaptada de Thiagarajan et al. (2006). Entende-se satisfação no trabalho como a percepção do funcionário de linha de frente quanto a sua situação atual, bem como a percepção que resulta do entendimento sobre os requerimentos exigidos para desenvolver aqueles processos de trabalho pelos quais ele é responsável (Tarrant, 2008). A escala de satisfação é constituída por 4 itens adaptados do trabalho de Tarrant (2008). O desempenho subjetivo em vendas refere-se à percepção subjetiva do funcionário quanto aos seus resultados de vendas e cumprimento das metas. A escala foi baseada nos indicadores de Verhoef e Leeflang (2009) e consistiu em 2 itens.

Todas as escalas são do tipo Likert e de 7 pontos, variando de $1=$ discordo totalmente até $7=$ concordo totalmente. Além do que, algumas covariáveis foram utilizadas como condições de controle, sendo: idade do funcionário, tempo de experiência no cargo atual, tempo de experiência na empresa e tempo de experiência do funcionário da linha de frente no ramo de atividade.

\section{Resultados}

Para a análise dos dados, primeiramente a variável comportamento ambidestro foi constituída a partir do produto das dimensões provisão de serviços e cross-/up-selling (Yu et al., 2013). As medidas descritivas estão apresentadas na Tabela 2. 
Tabela 2

Matriz de Correlação dos Construtos (Pearson)

\begin{tabular}{|c|c|c|c|c|c|c|c|c|c|c|c|}
\hline & Construtos & 1 & 2 & 3 & 4 & 5 & 6 & 7 & 8 & 9 & 10 \\
\hline 1 & Idade do funcionário & 1 & & & & & & & & & \\
\hline 2 & Tempo na Empresa & $0,65^{* *}$ & 1 & & & & & & & & \\
\hline 3 & Tempo no Cargo & $0,55^{* *}$ & $0,62^{* *}$ & 1 & & & & & & & \\
\hline 4 & Tempo no Ramo & $0,88^{* *}$ & $0,73^{* *}$ & $0,59^{* *}$ & 1 & & & & & & \\
\hline 5 & Conflito & 0,01 & 0,11 & 0,00 & 0,03 & 0,83 & & & & & \\
\hline 6 & Ambiguidade & 0,00 & $-0,08$ & $-0,06$ & $-0,06$ & 0,06 & 0,83 & & & & \\
\hline 7 & Sobrecarga & 0,05 & 0,03 & 0,09 & $-0,01$ & $-0,01$ & $0,12^{*}$ & 0,86 & & & \\
\hline 8 & Ambidestria $^{a}$ & $-0,05$ & 0,05 & $-0,02$ & 0,04 & $-0,04$ & $-0,198^{* *}$ & $-0,05$ & $0,84 / 0,75$ & & \\
\hline 9 & Satisf. no Trabalho & $-0,05$ & 0,02 & 0,02 & 0,08 & $-0,15^{*}$ & $-0,24^{* *}$ & $-0,09$ & $0,59^{* *}$ & 0,87 & \\
\hline & Desempenho & 0,00 & 0,04 & 0,05 & 0,07 & $-0,14^{*}$ & $-0,027^{* *}$ & $-0,05$ & $0,48^{* *}$ & $0,75^{* *}$ & 0,89 \\
\hline & Média & 35,62 & 9,21 & 4,92 & 14,91 & 3,62 & 3,12 & 4,95 & 38,81 & 5,44 & 5,27 \\
\hline & Desvio Padrão & 8,05 & 6,85 & 3,91 & 8,24 & 1,22 & 0,86 & 0,96 & 7,61 & 1,20 & 1,34 \\
\hline & $\mathrm{AVE}^{\mathrm{b}}$ & - & - & - & - & 0,49 & 0,53 & 0,64 & $0,56 / 0,48$ & 0,58 & 0,70 \\
\hline & $\mathrm{CR}^{\mathrm{c}}$ & - & - & - & - & 0,87 & 0,87 & 0,90 & $0,84 / 0,79$ & 0,85 & 0,82 \\
\hline & Raiz AVE & - & - & - & - & 0,70 & 0,73 & 0,80 & $0,75 / 0,70$ & 0,76 & 0,83 \\
\hline
\end{tabular}

Nota. Valores em itálico correspondem ao alfa de Cronbach.

aProvisão de Serviços/Cross-/Up-Selling, respectivamente; ${ }^{\mathrm{b}} \mathrm{AVE}=$ Variância Extraída Média; ${ }^{\mathrm{c}} \mathrm{CR}=$ Confiabilidade Composta. $* * p<0,01 ; * p<0,05$.

Para o exame das hipóteses, foram usadas as técnicas de análise de regressão múltipla e o teste de mediação de Hayes (2013). Os resultados dos efeitos diretos são apresentados na Tabela 3. As variáveis de controle, as três dimensões do estresse e o comportamento ambidestro explicam 37,9\% da variação da satisfação no trabalho e $25,9 \%$ do desempenho com vendas.

Tabela 3

\section{Efeitos Diretos da Ambidestria e do Estresse}

\begin{tabular}{lllll}
\hline Construtos Independentes & \multicolumn{2}{l}{ Satisfação no Trabalho } & \multicolumn{2}{l}{ Desempenho com Vendas } \\
\cline { 2 - 5 } & \multicolumn{1}{l}{ Beta } & $\boldsymbol{t}$ Valor & Beta & $\boldsymbol{t}$ Valor \\
\hline Idade do funcionário & $-0,284$ & $-2,881 * *$ & $-0,080$ & $-0,741$ \\
Tempo na Empresa & $-0,111$ & $-1,542$ & $-0,056$ & $-0,714$ \\
Tempo no Cargo & 0,022 & 0,365 & 0,041 & 0,612 \\
Tempo no Ramo & 0,370 & $3,395^{* *}$ & 0,128 & 1,073 \\
Conflito & $-0,112$ & $-2,394 *\left(\mathrm{H}_{1 \mathrm{~d}}\right)$ & $-0,114$ & $-2,237^{*}\left(\mathrm{H}_{1 \mathrm{a}}\right)$ \\
Ambiguidade & $-0,107$ & $-2,252^{*}\left(\mathrm{H}_{1 \mathrm{e}}\right)$ & $-0,164$ & $-3,153^{* *}\left(\mathrm{H}_{1 \mathrm{~b}}\right)$ \\
Sobrecarga & $-0,024$ & $-0,506\left(\mathrm{H}_{1 \mathrm{f}}\right)$ & 0,003 & $0,061\left(\mathrm{H}_{1 \mathrm{c}}\right)$ \\
Comportamento Ambidestro & 0,536 & $11,210^{* *}\left(\mathrm{H}_{2 \mathrm{~b}}\right)$ & 0,442 & $8,454^{* *}\left(\mathrm{H}_{2 \mathrm{a}}\right)$ \\
$\mathrm{R}^{2}$ Ajustado & 0,379 & & 0,259 & \\
$F$ (Modelo) & $23,232 * *$ & & $13,746 * *$ & \\
\hline
\end{tabular}

Nota. Os betas são os coeficientes padronizados de regressão. $* * p<0,01 ; * p<0,05$. 
As hipóteses iniciais pressupõem que o estresse de conflito, a ambiguidade e a sobrecarga de papéis possuem efeitos negativos na satisfação no trabalho e no desempenho. Os resultados do modelo de regressão demonstraram que o conflito possui uma relação negativa e significativa com satisfação $(\beta$ $=-0,112 ; p<0,05)$, suportando $\mathrm{H}_{1 \mathrm{~d}}$. Portanto, quanto mais o funcionário percebe suas atividades como conflitantes, menor é o seu grau de satisfação com o trabalho.

Do mesmo modo, a percepção de ambiguidade dos papéis também teve uma relação negativa com satisfação no trabalho $(\beta=-0,107 ; p<0,05)$, conforme esperado pela $\mathrm{H}_{1 \mathrm{e}}$. O funcionário, ao não compreender corretamente as expectativas sobre o seu trabalho devido à falta de informação, pode apresentar menores níveis de satisfação. Quanto à sobrecarga, apesar de o coeficiente de regressão evidenciar uma relação negativa como o esperado, o resultado é não significativo $(\beta=-0,024 ; p=\mathrm{NS})$, não permitindo suportar $\mathrm{H}_{1 \mathrm{f}}$.

Além do mais, os resultados do modelo de regressão salientaram que o conflito de papéis $(\beta=-$ $0,114 ; p<0,05)$ e a ambiguidade $(\beta=-0,164 ; p<0,01)$ explicam negativamente o desempenho com vendas, suportando as hipóteses $\mathrm{H}_{1 \mathrm{a}}$ e $\mathrm{H}_{1 \mathrm{~b}}$. Assim, quanto maior for a percepção de que as atividades realizadas pelo funcionário são conflitantes, e menor for a clareza de informações, menor é o resultado com vendas. Como nos resultados para a satisfação com o trabalho, a percepção de sobrecarga não demonstrou ser um fator determinante do desempenho com vendas $(\beta=0,003 ; p=\mathrm{NS})$, não permitindo sustentar $\mathrm{H}_{\mathrm{cc}}$.

A próxima hipótese indica que o comportamento ambidestro tem um efeito positivo na satisfação no trabalho. $\mathrm{O}$ resultado demonstra uma relação positiva e significativa $(\beta=0,536 ; p<0,01)$, conforme esperado pela $\mathrm{H}_{2 \mathrm{~b}}$. Dessa maneira, o funcionário, ao demonstrar capacidade de conciliar ambas as atividades de provisão de serviços e de cross-/up-selling, apresenta melhores níveis de satisfação. Exemplificando, ao prestar serviços de suporte ao cliente, o funcionário tem pleno conhecimento de suas características e necessidades, facilitando a oferta de vendas congruentes. Já a atividade de venda demanda conhecimento sobre os produtos, o que facilita também a prestação de serviços (Yu et al., 2013). A conciliação de ambas as atividades pode gerar benefícios ao funcionário sobre a melhor forma de aplicar seus recursos, o que torna o trabalho mais prazeroso.

A hipótese $\mathrm{H}_{2 \mathrm{a}}$ implica que o comportamento ambidestro tem um efeito positivo no desempenho com vendas. $\mathrm{O}$ resultado indicou uma relação positiva e significativa $(\beta=0,442 ; p<0,01)$, suportando $\mathrm{H}_{2 \mathrm{a}}$. Sendo assim, a capacidade de conciliar as atividades de suporte ao cliente e de vendas, além de melhorar a satisfação do funcionário com o seu trabalho, é convertida também em melhores resultados em vendas. Esses achados demonstram que o elemento mediador comportamento ambidestro tem relação direta com os dois elementos dependentes.

Para testar os efeitos mediados, adotou-se dois modos, o teste de Hayes (2013), com exame de bootstraping de 1.000 reamostras (modelo 4 do Process ${ }^{\circledR}$ ), e os testes de Sobel, Aroian e Goodman, com os coeficientes não padronizados e os erros padronizados. Os resultados são descritos na Tabela 4.

A ambiguidade possui um efeito direto e negativo na satisfação $(\beta=-0,182 ; p<0,01)$. Isso mostra que a falta de clareza na interpretação dos papéis de trabalho pelo funcionário pode ter um efeito prejudicial na satisfação no trabalho. Contudo, esse efeito também ocorre de forma indireta $\left(\beta_{\text {mediado }}=-\right.$ $0,155 ; p<0,01)$ quando há a variável mediadora ambidestria do funcionário. Portanto, a falta de compreensão sobre as expectativas da organização, como não saber qual atividade priorizar (prestar serviços ao cliente ou vender), tende a reduzir o nível de ambidestria do funcionário, o que, por sua vez, reduz o nível de satisfação com o trabalho. Esse resultado indireto suporta a hipótese $\mathrm{H}_{3 \mathrm{a}}(\mathrm{Z}=-3,380$; $p<0,01)$ e é congruente com Bonesso, Gerli e Scapolan (2014).

O mesmo processo de mediação ocorre com o desempenho com vendas. A ambiguidade de papéis possui um impacto direto e negativo no desempenho com vendas $(\beta=-0,291 ; p<0,01)$. Entretanto, há um efeito indireto mediado pelo comportamento ambidestro $\left(\beta_{\text {mediado }}=-0,136 ; p<0,01 ; \mathrm{Z}=-3,259 ; p<\right.$ 0,01 ), o que suporta a hipótese $\mathrm{H}_{3 \mathrm{~b}}$. Diante desse achado, a percepção de ambiguidade de papéis tem efeito negativo sobre a ambidestria, impactando também negativamente o desempenho com vendas. 
Tabela 4

Efeitos do Estresse Mediados pelo Comportamento Ambidestro

\begin{tabular}{lllllll}
\hline & \multicolumn{2}{l}{ Satisfação no Trabalho } & \multicolumn{3}{l}{ Desempenho com Vendas } \\
\cline { 2 - 7 } & Conflito & Ambiguidade & Sobrecarga & Conflito & Ambiguidade & Sobrecarga \\
\hline Efeito Direto & $-0,120^{* *}$ & $-0,182^{* *}$ & $-0,078$ & $-0,135^{*}$ & $-0,291^{* *}$ & $-0,044$ \\
Efeito Mediado & $-0,023$ & $-0,155^{* *}$ & $-0,034$ & $-0,021$ & $-0,136^{* *}$ & $-0,031$ \\
Z (Hayes, 2013) & $-0,712$ & $-3,380^{* *}$ & $-0,821$ & $-0,709$ & $-3,259^{* *}$ & $-0,818$ \\
Sobel & $-0,715$ & $-3,404^{* *}$ & $-0,824$ & $-0,714$ & $-3,268^{* *}$ & $-0,822$ \\
Aroian & $-0,713$ & $-3,394^{* *}$ & $-0,821$ & $-0,710$ & $-3,249^{* *}$ & $-0,817$ \\
Goodman & $-0,717$ & $-3,414^{* *}$ & $-0,826$ & $-0,718$ & $-3,287^{* *}$ & $-0,827$ \\
\hline
\end{tabular}

Os efeitos do conflito $\left(\mathrm{H}_{4 \mathrm{a}-\mathrm{b}}\right)$ e da sobrecarga $\left(\mathrm{H}_{5 \mathrm{a}-\mathrm{b}}\right)$ de papéis mediados pelo comportamento ambidestro não apresentaram resultados significativos, não permitindo suportar as hipóteses (ver tabela 4). Duas explicações são lançadas para explicar as hipóteses não suportadas.

Primeiro, a ambidestria consiste na habilidade em perseguir objetivos diferentes ou conflitantes ao mesmo tempo (Gibson \& Birkinshaw, 2004). As atividades de prestação de serviços e de vendas cross-/up-selling são conflitantes em termos de objetivos, eficiência e receita, respectivamente (Rust, Moorman, \& Dickson, 2002). Enquanto a percepção de ambiguidade gera uma redução no nível de ambidestria, reduzindo o desempenho do funcionário, a percepção de conflito e a sobrecarga de trabalho não exercem o mesmo efeito. A inexistência das influências não permite haver a transmissão de um efeito indireto.

Segundo, os objetivos conflitantes causam uma percepção de sobrecarga, o que leva os funcionários a enfrentarem trade-offs sobre a melhor forma de aplicar recursos (ex.: tempo). A ambidestria prevê a capacidade do funcionário em decidir sobre os trade-offs e a melhor forma para empregar os recursos entre os objetivos conflitantes de forma a garantir um melhor resultado (Gibson \& Birkinshaw, 2004; Jasmand et al., 2012). Nesse contexto, a sobrecarga de trabalho pode gerar um conflito grande que não auxilia o sujeito a decidir sobre os trade-offs e a implementar a ambidestria.

\section{Considerações Finais}

Esta seção é destinada a apresentar as conclusões teóricas, as aplicações gerenciais e as sugestões de pesquisas futuras deste estudo. Sobre as contribuições teóricas, três contribuições à literatura são apresentadas. Primeiro, a pesquisa propôs que o comportamento ambidestro do funcionário da linha de frente medeia a relação entre a ambiguidade e as medidas de desempenho com vendas e satisfação no trabalho. De acordo com a teoria da ambidestria organizacional, a sobrevivência ou prosperidade depende da capacidade em conciliar atividades com objetivos distintos e que competem entre elas por recursos (March, 1991). As agências bancárias, ao exigirem de seus funcionários da linha de frente que prestem serviços aos clientes, ao mesmo tempo em que devem procurar oportunidade de vendas, podem criar um ambiente de pressão sobre os funcionários, o que, por sua vez, segundo a teoria de estresse de papel, pode prejudicar o desempenho (Kahn et al., 1964).

Os resultados demonstram que o efeito negativo da percepção de ambiguidade de papéis sobre as medidas de desempenho (satisfação no trabalho e desempenho com vendas) é mediado pelo comportamento ambidestro do funcionário. A ambidestria demanda que o funcionário busque objetivos conflitantes, realize atividades contraditórias e desenvolva competências distintas (Jasmand et al., 2012). Portanto, é um contexto que pode causar confusão no funcionário sobre quais papéis são 
esperados que ele realize. Quando há incongruência entre as expectativas sobre o funcionário e comportamento por ele desempenhado, em outras palavras, quando não há clareza nas informações repassadas aos funcionários sobre as atividades que são esperadas que eles realizem, há uma tendência maior de insatisfação no trabalho e resultados piores (Bonesso et al., 2014). Desse modo, a falta de informação para o trabalho, ou a falha de compreensão dela, pode prejudicar a adoção do comportamento ambidestro, reduzindo o desempenho do funcionário.

Sobre os efeitos mediados do conflito de papéis e da sobrecarga de trabalho sobre as medidas de desempenho, os resultados não foram significativos. O conflito de papéis pressupõe que o funcionário considera contraditório o objetivo sobre o seu trabalho (Tarrant, 2008). A busca por objetivos contraditórios pode ser compensada com a adoção de sistemas de remuneração variável, planos de carreira ou outros benefícios sociais (William \& Alliger, 1994), tornando-se um estado psicológico positivo do estresse e de desafio aos funcionários (McGowan, Gardner, \& Fletcher, 2006).

Não obstante, a ambidestria pressupõe também trade-offs sobre a melhor forma de aplicar os recursos entre as atividades de serviços e vendas (Jasmand et al., 2012). O funcionário deve demonstrar a capacidade de tomar a melhor decisão sobre a forma de aplicar os recursos entre as atividades conflitantes. A sobrecarga de papéis aumenta o problema de trade-off, tornando o tempo de trabalho mais escasso devido à percepção de inconsistência entre o tempo disponível e o tempo necessário para realizar tarefas (Yongkang et al., 2014). O funcionário deve escolher uma estratégia que lhe permita atender os objetivos conflitantes simultaneamente (Orehek \& Nieuwenhuis, 2013). Assim sendo, como no caso do conflito de papéis, a sobrecarga, por si só, não foi determinante para influenciar negativamente a adoção do comportamento ambidestro neste estudo.

Segundo, este estudo propôs investigar os efeitos diretos do estresse nas medidas de desempenho. Conforme a teoria do estresse de papel, quando o funcionário não tem diretrizes claras quanto às suas responsabilidades, ele experimentará o estresse, tornando-se menos satisfeito e menos eficaz (Idris, 2011). Esperava-se que as três dimensões do estresse de papel (ambiguidade, conflito e sobrecarga) apresentassem efeitos negativos e significativos. Duas hipóteses foram suportadas, exceto a hipótese referida à percepção da sobrecarga de papéis. Assim, a falta de clareza do funcionário sobre as expectativas da organização e a percepção de que os objetivos demandados são conflitantes se caracterizam como uma situação de estresse que pode afetar negativamente o desempenho.

Entretanto, neste trabalho, a percepção de incongruência entre as demandas de trabalho e os recursos disponíveis (ex.: tempo), apesar de gerar estresse, por si só, não são fatores negativos determinantes do resultado. Em outras palavras, há funcionários que lidam bem com o contexto de incongruência por meio de motivações intrínsecas e uma forte orientação para a ação ou por meio de recompensas e incentivos, como sistemas de remuneração variável, comuns no meio bancário, como propõe a teoria do modo regulatório (Kruglanski et al., 2000). Ademais, a incongruência pode ser vista como um elemento do eustresse (McGowan et al., 2006), desafiando e estimulando o funcionário a desenvolver um comportamento ambidestro. Os funcionários necessitam de um nível moderado de estresse para permanecerem alertas e capazes de atuar de forma eficiente nas organizações (Kumar, 2012).

Terceiro, apesar da constatação dos efeitos negativos do estresse nas medidas de desempenho, a pesquisa demonstrou que o impacto positivo do comportamento ambidestro é maior. Tanto para a satisfação no trabalho quanto para o desempenho com vendas, o efeito foi positivo e significativo. Ao prestar serviços de suporte, o funcionário tem acesso às diversas informações sobre o cliente, como histórico de compras e preferências e domínio sobre os produtos. Assim, há uma forte orientação para o cliente, facilitando a oferta de produtos congruentes e contribuindo com o resultado de vendas (Verbeke et al., 2011). Do mesmo modo, a prestação de um serviço de modo satisfatório pode gerar confiança e uma avaliação favorável do cliente, fatores que podem aumentar a intenção de compra e as vendas.

Além das contribuições teóricas, os resultados desta pesquisa oferecem contribuições gerenciais aos bancos que demandam dos seus funcionários de linha de frente um comportamento ambidestro. A exigência de atividades conflitantes pode gerar no funcionário estresse de trabalho, percebido como 
conflito, ambiguidade e sobrecarga de papéis. Observa-se que o estresse de papel possui um efeito negativo nos resultados do funcionário de linha de frente. Na medida em que práticas e procedimentos organizacionais se caracterizam pela falta de preocupação com o fluxo adequado de comunicação e com o bem-estar do empregado, pode-se criar um contexto ambíguo e contraditório, prejudicando o desempenho individual. Portanto, os bancos devem cuidar para que os funcionários compreendam corretamente as funções e metas que são esperadas que eles desempenhem, assim como compreendam os benefícios a eles atribuídos quando do cumprimento dessas metas.

Ademais, destaca-se que a ambiguidade de papéis possui também um efeito indireto nas medidas de desempenho via comportamento ambidestro, enquanto conflito e sobrecarga de papéis não apresentaram o mesmo resultado. Sendo assim, o foco maior dos bancos pode ser no treinamento dos funcionários para que compreendam com clareza aquilo que lhe foi demandado. Os gerentes de agência, além de se comunicarem bem com os funcionários, devem se certificar que esses últimos compreenderam o que é esperado que eles realizem e como devem fazer. Atividades como a divulgação e acompanhamento constante de metas, o direcionamento e monitoramento das ações por parte dos gerentes e a realização de feedbacks sobre o desempenho atingido contribuem para uma melhor compreensão do funcionário sobre as expectativas da organização, reduzindo a percepção de ambiguidade.

Em contrapartida ao efeito negativo da ambiguidade sobre as medidas de desempenho, os impactos da percepção de conflito entre as atividades e a sobrecarga de trabalho podem ser minimizados quando o funcionário demonstra o comportamento ambidestro, ou seja, uma capacidade de lidar com esse contexto. O efeito positivo do comportamento ambidestro sobre as medidas de desempenho é maior do que os efeitos negativos das dimensões do estresse. Sendo assim, processos de recrutamento e seleção que visem identificar o comportamento ambidestro do candidato podem nutrir a organização com funcionários capazes de assimilar maiores conteúdos e informações de diversas fontes, colaborando para a melhoria do clima organizacional. No mesmo sentido, programas de treinamento podem direcionar suas atividades na busca por desenvolver características de ambidestria no comportamento dos seus funcionários, como orientá-los sobre as maneiras de explorar as complementaridades das atividades de serviços e vendas, conforme sugerido por Yu et al. (2013).

Por fim, o banco deve compreender as orientações motivacionais de seus funcionários para encontrar o melhor ajustamento entre as características individuais e os sistemas de avaliação e recompensa, por exemplo, entendendo o grau em que os funcionários atribuem importância ao reconhecimento social e recompensas em dinheiro (ver Giacomantonio, Mannetti, \& Pierro, 2013).

Em relação às sugestões de pesquisas futuras, inicialmente, destaca-se que algumas relações esperadas não apresentaram resultados significativos. A sobrecarga de papéis não foi determinante para impactar o desempenho do funcionário, seja de forma direta, seja de forma indireta (mediada pelo comportamento ambidestro). Do mesmo modo, o conflito de papéis também não apresentou efeito mediado significativo no desempenho. Esses resultados sugerem a possibilidade de novas variáveis que interferem nessa relação. Por exemplo, sob quais circunstâncias de trabalho (ex.: baixa/alta remuneração) a sobrecarga de trabalho e a percepção de conflito passam a ser prejudiciais ao desempenho ou ao comportamento ambidestro?

Funcionários podem aceitar papéis de estresse em troca de benefícios sociais ou monetários (Idris, 2011). Todavia, há evidências empíricas que os indivíduos apresentam orientações motivacionais distintas, como sugerido pela teoria do modo regulatório (Kruglanski et al., 2000), e que, por isso, responderão de forma distinta a esses benefícios (Giacomantonio et al., 2013). Estudos futuros podem investigar em que contexto a relação entre estresse de trabalho e medidas de desempenho pode ser alterada, em função dos sistemas de avaliação, de benefícios e das orientações de autorregulação de cada funcionário.

Além disto, conflito e sobrecarga de papéis em um ambiente de exigência do comportamento ambidestro podem ser considerados estados psicológicos positivos do estresse, denominado euestresse (McGowan et al., 2006). Ao invés de serem vistos como elementos ameaçadores e danosos aos 
funcionários em temos de bem-estar no trabalho e de problemas físicos e psicológicos, estes elementos do eustresse podem ser tratados como desafiadores diante de uma tarefa difícil e estão associados a índices positivos de saúde, bem-estar e satisfação no trabalho (McGowan et al., 2006). Estudos futuros podem trabalhar nessa linha de raciocínio.

\section{Referências}

Aghdasia, S., Kiamaneshb, A. R., \& Ebrahim, A. N. (2011). Emotional intelligence and organizational commitment: testing the mediatory role of occupational stress and job satisfaction. ProcediaSocial and Behavioral Sciences, 29(1), 1965-1976. http://dx.doi.org/10.1016/j.sbspro.2011.11.447

Ahsan, N., Abdullah, Z., Fie, D. G., \& Alam, S. S. (2009). A study of job stress on job satisfaction among university staff in Malaysia: empirical study. European Journal of Social Sciences, 8(1), 121-131.

Behrman, D. N., \& Perreault, W. D. (1982). Measuring the performance of industrial salespersons. Journal of Business Research, 10(3), 355-370. http://dx.doi.org/10.1016/0148-2963(82)90039-X

Benner, M. J., \& Tushman, M. L. (2003). Exploitation, exploration, and process management: the productivity dilemma revisited. Academy of Management, 28(2), 238-256. http://dx.doi.org/10.5465/AMR.2003.9416096

Bonesso, S., Gerli, F., \& Scapolan, A. (2014). The individual side of ambidexterity: do individuals' perceptions match actual behaviors in reconciling the exploration and exploitation trade-off?. European Management Journal, 32(3), 392-405. http://dx.doi.org/10.1016/j.emj.2013.07.003

Cass, M. H., Siu, O. L., Faragher, E. B., \& Cooper, C. L. (2003). A meta-analysis of the relationship between job satisfaction and employee health in Hong Kong. Stress and Health, 19(2), 79-95. http://dx.doi.org/10.1002/smi.959

Chida, Y., Hamer, M., Wardle, J., \& Steptoe, A. (2008). Do stress-related psychosocial factors contribute to cancer incidence and survival? Nature Clinical Practice Oncology, 5(8), 466-475. http://dx.doi.org/10.1038/ncponc1134

Claessens, B. J., Van Eerde, W., Rutte, C. G., \& Roe, R. A. (2004). Planning behavior and perceived control of time at work. Journal of Organization Behavior, 25(8), 937-950. http://dx.doi.org/10.1002/job.292

Deery, S., Roderick, I., \& Walsh, J. (2002).Work relationships in telephone call centres: understanding emotional exhaustion and employee withdrawal. Journal of Management Studies, 39(4), 471-496. http://dx.doi.org/10.1111/1467-6486.00300

Duncan, R. B. (1974). The ambidextrous organization: designing dual structures for innovation. In R. H. Kilman, L. R. Pondy, \& D. P. Slevin (Eds.), The management of organization design: strategies and implementation (Vol. 1, pp. 167-188). New York: University of Pittsburgh.

Dutta, S. K. (2013). Ambidexterity as a mediating variable in the relationship between dynamism in the environment, organizational context and strategic renewal. Jindal Journal of Business Research, 2(1), 27-41. http://dx.doi.org/10.1177/2278682114533177

Ford, M. T., Cerasoli, C. P., Higgins, J. A., \& Decesare, A. L. (2011). Relationships between psychological, physical, and behavioural health and work performance: a review and metaanalysis. Work \& Stress, 25(3), 185-204. http://dx.doi.org/10.1080/02678373.2011.609035 
Giacomantonio, M., Mannetti, L., \& Pierro, A. (2013). Locomoting toward well-being or getting entangled in a material world: regulatory modes and affective well-being. Journal of Economic Psychology, 38, 80-89. http://dx.doi.org/10.1016/j.joep.2012.07.003

Gibson, C., \& Birkinshaw, J. (2004). The antecedents, consequences, and mediating role of organizational ambidexterity. Academy of Management Journal, 47(2), 209-226. http://dx.doi.org/10.2307/20159573

Gilsing, V. A. (2002). Co-evolution of exploration \& exploitation in a sectoral system of innovation. Proceedings of the PhD Conference, Aalborg, Denmark. Recuperado de http://www.druid.dk/conferences/winter2002/gallery/gilsing.pdf

Günes, E. D., Aksin, O. Z., Örmeci, L., \& Özden, S. H. (2010). Modeling customer reactions to sales attempts: if cross-selling backfires. Journal of Service Research, 13(2), 168-83. http://dx.doi.org/10.1177/1094670509352677

Hayes, A. F. (2013). Introduction to mediation, moderation, and conditional process analysis: a regression-based approach. New York: Guilford Press.

Heikkilä, K., Nyberg, S. T., Theorell, T., Fransson, E. I., Alfredsson, L., Bjorner, J. B., \& Dragano, N. (2013). Work stress and risk of cancer: meta-analysis of 5700 incident cancer events in 116000 European men and women. British Medical Journal, 346, 65. http://dx.doi.org/10.1136/bmj.f165

Idris, K. M. (2011). Over time effects of role stress on psychological strain among malaysian public university academics. International Journal of Business and Social Science, 2(9), 154-161. Recuperado de http://ijbssnet.com/journals/Vol__2_No._9_[Special_Issue_-_May_2011]/19.pdf

Jansen, J. J. P., Bosch, V. D., \& Volberda, H. W. (2006). Exploratory innovation, exploitative innovation, and performance: effects of organizational antecedents and environmental moderators. Management Science, 52(11), 1661-1674. http://dx.doi.org/10.1287/mnsc.1060.0576

Jasmand, C., Blazevic V., \& Ruyter, K. (2012). Generating sales while providing service: a study of customer service representatives ambidextrous behavior. Journal of Marketing, 76(1), 20-37. http://dx.doi.org/10.1509/jm.10.0448

Kahn, R. L., Wolfe, D. M., Quinn, R. P., \& Snoek, J. D. (1964). Organizational stress: studies in role conflict and ambiguity. New York: Wiley.

Katz, D., \& Kahn, R. (1966). The social psychology of organizations. New York: Wiley.

Kortmann, S., Gelhard, C., Zimmermann, C., \& Piller, F. T. (2014). Linking strategic flexibility and operational efficiency: the mediating role of ambidextrous operational capabilities. Journal of Operations Management, 32(7), 475-490. http://dx.doi.org/10.1016/j.jom.2014.09.007

Kruglanski, A. W., Thompson, E. P., Higgins, E. T., Atash, M., Pierro, A., Shah, J. Y., \& Spiegel, S. (2000). To "do the right thing" or to "just do it": locomotion and assessment as distinct selfregulatory imperatives. Journal of Personality and Social Psychology, 79(5), 793-815. http://dx.doi.org/10.1037/0022-3514.79.5.793

Kumar, A. S. (2012). A critical analysis of stress faced by teaching professionals at management institutes in Pune with special references to role stressors and job satisfaction (Doctoral thesis). Tilak Maharashtra University, Pune, Indoa. Recuperado de http://shodhganga.inflibnet.ac.in/handle/10603/4401

Lambert, V., \& Lambert, C. (2001). Literature review of role stress/strain on nurses: an international perspective. Nursing and Health Science, 3(3), 161-172. http://dx.doi.org/10.1046/j.14422018.2001.00086.x 
Le Fevre, M., Matheny, J., \& Kolt, G. S. (2003). Eustress, distress, and interpretation in occupational $\begin{array}{lllll}\text { stress. Journal of Managerial Pychology, 18(7), } & \text { 726-744. }\end{array}$ http://dx.doi.org/10.1108/02683940310502412

Li, Y. H., \& Huang, J. W. (2012). Ambidexterity's mediating impact on product development proficiency and new product performance. Industrial Marketing Management, 41(7), 1125-1132. http://dx.doi.org/10.1016/j.indmarman.2012.05.002

March, J. G. (1991). Exploration and exploitation in organizational learning. Organization Science, 2(1), 71-87. http://dx.doi.org/10.1287/orsc.2.1.71

McGowan, J., Gardner, D., \& Fletcher, R. (2006). Positive and negative affective outcomes of occupational stress. New Zealand Journal of Psychology, 35(2), 92-98. Recuperado de http://connection.ebscohost.com/c/articles/22462768/positive-negative-affective-outcomesoccupational-stress

Mom, T. J. M., Van Den Bosch, F. A. J., \& Volberda, H. W. (2009). Understanding variation in managers' ambidexterity: investigating direct and interaction effects of formal, structural and personal coordination mechanisms. Organization Science, 20(4), 812-28. http://dx.doi.org/10.1287/orsc.1090.0427

Muchinsky, P. M. (2000). Psychology applied to work (6th ed.). Belmont: Wadsworth.

Nevo, B. (1985). Face validity revisited. Journal of Educational Measurement, 22(4), 287-293. http://dx.doi.org/10.1111/j.1745-3984.1985.tb01065.x

Orehek, E., \& Vazeou-Nieuwenhuis, A. (2013). Sequential and concurrent strategies of multiple goal pursuit. Review of General Psychology, 17(3), 339-349. http://dx.doi.org/10.1037/a0032584.

Patterson, P., Yu, T., \& Kimpakorn, N. (2014). Killing two birds with one stone: cross-selling during service delivery. Journal of Business Research, 67(9), 1944-1952. http://dx.doi.org/10.1016/j.jbusres.2013.11.013

Pinheiro, F. A., \& Günther, I. A. (2002). Estresse ocupacional e indicadores de saúde em gerentes de um banco estatal. Revista Psicologia: Organização e Trabalho, 2(2), 65-84. Recuperado de https://periodicos.ufsc.br/index.php/rpot/article/view/6848/6329

Posig, M., \& Kickul, J. (2003). Extending our understanding of burnout: test of an integrated model in nonservice occupations. Journal of Occupational Health Psychology, 8(1), 3-19. http://dx.doi.org/10.1037/1076-8998.8.1.3

Raisch, S., Birkinshaw, J., Probst, G., \& Tushman, M. L. (2009) Organizational ambidexterity: balancing exploitation and exploration for sustained performance. Organization Science, 20(4), 685-695. http://dx.doi.org/10.1287/orsc.1090.0428

Rizzo, J., R., House, R., \& Lirtzman, L. (1970). Role conflict and ambiguity in complex organizations. Administrative Science Quarterly, 15(1), 150-63. http://dx.doi.org/10.2307/2391486

Rust, R. T., Moorman, C., \& Dickson, P. R. (2002). Getting return on quality: revenue expansion, cost reduction, or both? Journal of Marketing, 66(4), 7-24. http://dx.doi.org/10.1509/jmkg.66.4.7.18515

Sarkees, M., Hulland, J., \& Prescott, J. (2010). Ambidextrous organizations and firm performance: the role of marketing function implementation. Journal of Strategic Marketing, 18(2), 165-184. http://dx.doi.org/10.1080/09652540903536982

Simsek, Z. (2009). Organizational ambidexterity: towards a multilevel understanding. Journal of Management Studies, 46(4), 597-624. http://dx.doi.org/10.1111/j.1467-6486.2009.00828.x 
Singh, J. (1998). Striking a balance in boundary spanning positions: an investigation of some unconventional influences of role stressors and job characteristics on job outcomes of salespeople. Journal of Marketing, 62(3), 69-86. http://dx.doi.org/10.2307/1251744

Tarrant, T. A. (2008). Role conflict, role ambiguity and job satisfaction of the nurse executive (Doctoral dissertation). University of Nevada, Division of Health Sciences, Las Vegas.

Thiagarajan, P., Chakrabarty, S., \& Taylor, R. (2006). A confirmatory factor analysis of Reilly's Role Overload Scale. Educational \& Psychological Measurement, 66(4), 657-666. http://dx.doi.org/10.1177/0013164405282452

Van der Borgh, M., \& Schepers, J. J. (2014). Do retailers really profit from ambidextrous managers? The impact of frontline mechanisms on new and existing product selling performance. Journal of Product Innovation Management, 31(4), 710-727. http://dx.doi.org/10.1111/jpim. 12158

Verbeke, W., Dietz, B., \& Verwaal, E. (2011). Drivers of sales performance: a contemporary metaanalysis. Have salespeople become knowledge brokers? Journal of the Academy Marketing Science, 39(3), 407-428. http://dx.doi.org/10.1007/s11747-010-0211-8

Verhoef, P. C., \& Leeflang, P. S. H. (2009). Understanding the marketing department's influence within the firm. Journal of Marketing, 73(2), 14-37. http://dx.doi.org/10.1509/jmkg.73.2.14

William, K. J., \& Alliger, G. M. (1994). Role stressors, mood spillover, and perceptions of work-family conflict in employed parents. Academy of Management Journal, 37(4), 837-868. http://dx.doi.org/10.2307/256602

Yavas, U., Karatepe, O. M., Avci, T., \& Tekinkus, M. (2003). Antecedents and outcomes of service recovery performance: an empirical study of frontline employees in Turkish banks. International Journal of Bank Marketing, 21(5), 255-265. http://dx.doi.org/10.1108/02652320310488439

Yongkang, Z., Weixi, Z., Yalin, H., Yipeng, X., \& Liu, T. (2014). The relationship among role conflict, role ambiguity, role overload and job stress of Chinese middle- level cadres. Journal Article Chinese Studies, 3(1), 8-11. http://dx.doi.org/10.4236/chnstd.2014.31003

Yu, T., Patterson, P. G., \& Ruyter. K. (2013). Achieving service-sales ambidexterity. Journal of Service Research, 16(1), 52-66. http://dx.doi.org/10.1177/1094670512453878

Yu, T., Patterson, P., \& de Ruyter, K. (2015). Converting service encounters into cross-selling opportunities: does faith in supervisor ability help or hinder service-sales ambidexterity? European Journal of Marketing, 49(3/4), 491-511. http://dx.doi.org/10.1108/EJM-10-2013-0549

\title{
Dados dos Autores
}

\author{
Valter Afonso Vieira \\ Av. Colombo, 5790, 86700-000, Maringá, PR, Brasil. E-mail: valterafonsovieira@ gmail.com \\ Marco Aurelio Garcia Rosa \\ Av. Colombo, 5790, 86700-000, Maringá, PR, Brasil. E-mail: marcogarcia81 @ hotmail.com \\ Valter da Silva Faia \\ Av. Colombo, 5790, 86700-000, Maringá, PR, Brasil. E-mail: valterfaia@gmail.com
}

